\title{
Topographic association between active gastritis and Campylobacter pylori colonisation
}

\author{
E BAYERDÖRFFER,* H OERTEL, ${ }^{*}$ NLEHN, $\dagger$ G KASPER, $\dagger$ G A MANNES,* \\ T SAUERBRUCH, * M STOLTE $\ddagger$
}

From the *Medical Department II, Klinikum Grosshadern, University of Munich, the †Institute for Microbiology, Municipal Hospital Bogenhausen, Munich, and the $\ddagger$ Institute for Pathology, Klinikum Bayreuth, Bayreuth, West Germany

SUMMARY One thousand biopsy specimens obtained from 10 sites in the stomachs of 50 patients were examined for the presence of active chronic gastritis and Campylobacter pylori. All 32 patients with active chronic gastritis at 234 out of 320 sites were positive for $C$ pylori: 227 showed colonisation with $C$ pylori by the Warthin-Starry stain; and 222 were positive by culture. $C$ pylori was not found in 18 patients with inactive chronic gastritis or histologically normal mucosa. The area of $C$ pylori colonisation was larger than the area of active chronic gastritis in 289 positive specimens on culture and 261 on staining, respectively, suggesting that $C$ pylori colonisation may precede the development of active chronic gastritis.

It is concluded that patchy distribution of active chronic gastritis and $C$ pylori colonisation must be considered, particularly in serology or breath test studies where the histological examination serves as a reference. Furthermore, it may have important implications for the follow up of patients after antibacterial treatment. The topographic and specific association of $C$ pylori and active chronic gastritis provides further evidence for the pathogenic role of $C$ pylori in active chronic gastritis.

Evidence has been accumulating for a primary pathogenic role for Campylobacter pylori in the development of active chronic gastritis. ${ }^{1-9}$ This evidence has been challenged. A major argument against this is that it is also isolated from inactive chronic gastritis ${ }^{29-14}$ and from histologically normal mucosa. ${ }^{214}$ The association between $C$ pylori and active chronic gastritis has been reported to range from $82-97 \%$ in selected patients with duodenal ulcer or non-ulcer dyspepsia. ${ }^{20-14}$.

These unequivocal results on the association between $C$ pylori and active chronic gastritis or other types of gastritis may be explained by either methodological shortcomings or an unspecific association between $C$ pylori and active chronic gastritis and other types of gastritis.

As early work suggested that a considerable number of patients may have a patchy distribution of gastritis ${ }^{15}$ we designed this study to analyse further the sensitivity of endoscopic biopsies for detecting active chronic gastritis in the antrum and body of the stomach and its topographic association with $C$ pylori detected by Warthin-Starry stain and culture.

Accepted for publication 16 March 1989

\section{Patients and methods}

Fifty consecutive patients attending our endoscopy unit for examination of the upper gastrointestinal tract were included in the study. Patients taking antibiotics or bismuth, and those with a bleeding ulcer or an acute haemorrhage from other sites in the upper gastrointestinal tract, or with any carcinoma were excluded. All patients gave their informed consent.

In accordance with a standardised protocol, 20 biopsy specimens were taken at 10 defined sites, beginning in the antrum and proceeding towards the cardia. One of two biopsy specimens from each site was stained with haematoxylin and eosin to grade the gastritis, and by Warthin-Starry stain to detect Cpylori. The corresponding 10 specimens were used to culture $C$ pylori and to prepare smears.

Biopsies were always performed in the same sequence and coded with random numbers in groups of two patients.

The biopsy specimens were placed in Port-A-Cul medium (Becton Dickinson, Heidelberg) for microbiological examination and cultured within four hours. Mucosal specimens were smeared on a sterile slide using a sterile scalpel and subsequently spread on culture plates. 
$C$ pylori was cultured in parallel on Wilkins Chalgren anaerobe agar (Oxoid, London) containing $10 \%$ human erythrocytes and Skirrow's Campylobacter antibiotic supplement, and additionally on chocolate agar (Blood Agar Base No 2, Oxoid) containing $10 \%$ horse serum (added at $50^{\circ} \mathrm{C}$ ). Incubation and microscopic and biochemical identification of $C$ pylori were performed as described elsewhere. ${ }^{16}$

After drying, the smear was stained with concentrated fuchsin. The culture was positive when at least one colony of $C$ pylori was identified. Each stain was examined for at least 10 minutes and taken as positive when at least one typical organism was found.

Biopsy specimens were fixed immediately with $3.7 \%$ neutral formalin for histological examination. Thin sections were routinely prepared and stained with haematoxylin and eosin and the Warthin-Starry technique. Gastritis was graded from 1 to 4 (grades 1 to 3 for superficial gastritis and 4 for superficial gastritis with mild atrophy of the glands with modifications according to Whitehead. ${ }^{17}$ The presence of neutrophils was diagnostic of active chronic gastritis. Chronic inflammatory changes with no polymorphonuclear infiltration were graded as chronic gastritis. Each stain was examined for at least five minutes for the presence of $C$ pylori or neutrophils.

A patient positive for $C$ pylori and active chronic gastritis in at least one biopsy specimen originating from the same biopsy site was judged to have $C$ pylori positive active chronic gastritis.

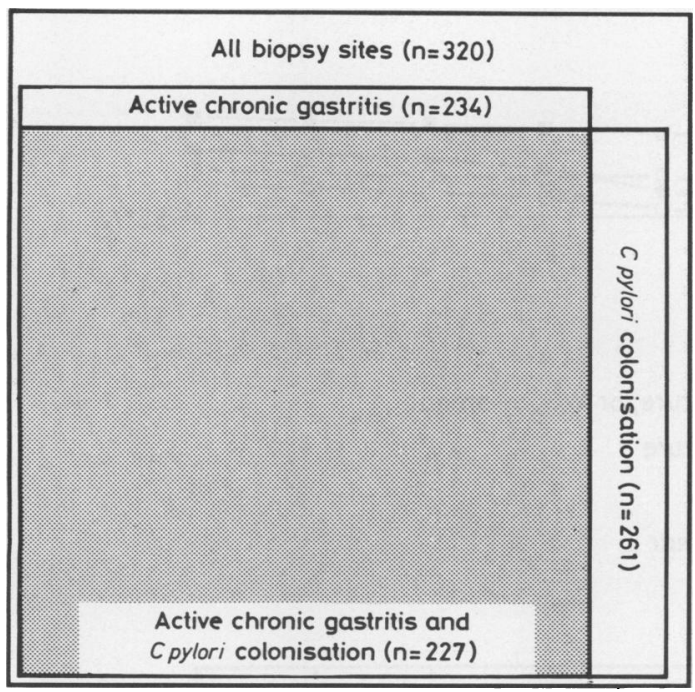

Fig 1 Area of active chronic gastritis $(n=234)$, area of colonisation with $C$ pylori shown by Warthin-Starry stain $(n=261)$, area of active chronic gastritis and $C$ pylori colonisation $(n=227)$ in 32 patients with 320 biopsy sites.
Specimens examined microbiologically and histologically were assumed to be from identical sites for statistical analysis. We assumed that the detection of $C$ pylori in one biopsy specimen was an independent event (urn model with putting sample back). The events " $C$ pylori colonisation" and "no $C$ pylori colonisation" are mutually exclusive: $\mathrm{f}(C$ pylori, no $C$ pylori $)=0$.

We selected the probability of the event " $C$ pylori" occurring $\mathrm{x}$ times $(\mathrm{x}=0,1,2,3 \ldots \mathrm{n})$ in $\mathrm{n}$ random samples. In mutually exclusive events the probability that in $\mathrm{n}$ independent random experiments the positive

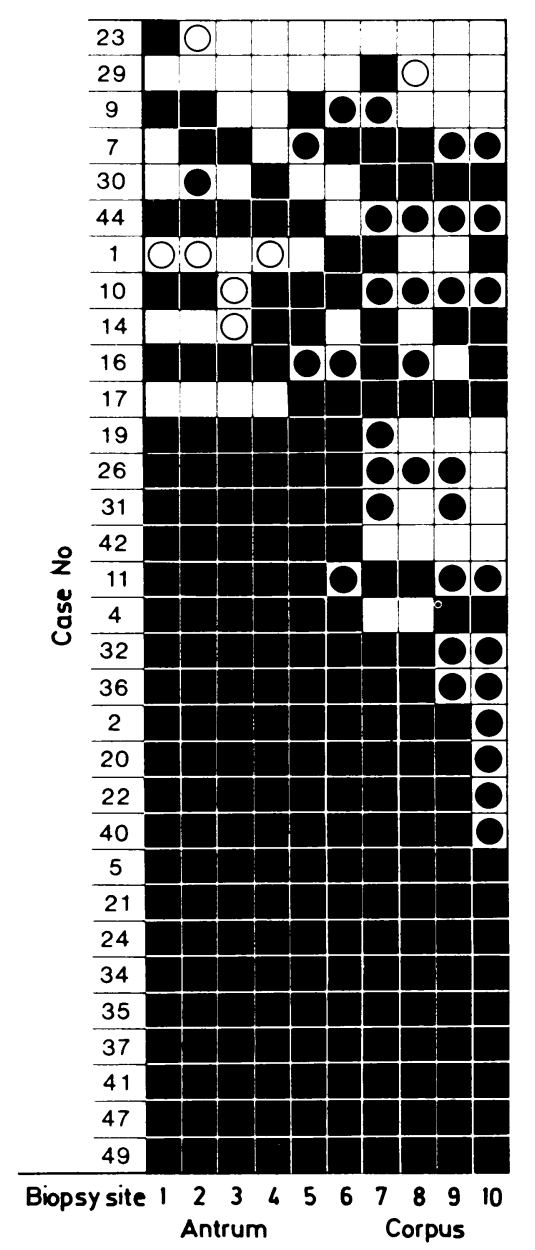

Fig 2 Patchy distribution of active chronic gastritis and C pylori colonisation in 32 patients with active chronic gastritis at one or more sites. Biopsy sites with $C$ pylori positive active chronic gastritis; $\bigcirc=C$ pylori negative active chronic gastritis; $=C$ pylori positive sites with no active chronic gastritis; $\square=$ biopsy sites showing neither active chronic gastritis nor $C$ pylori colonisation. 
Table Diagnoses of patients studied in per cent

\begin{tabular}{|c|c|c|c|c|c|c|c|c|c|c|}
\hline & \multicolumn{10}{|c|}{ No of biopsies } \\
\hline & 1 & .2 & 3 & 4 & 5 & 6 & 7 & 8 & 9 & 10 \\
\hline $\begin{array}{l}\text { ACG, CP + } \\
\text { ACG, CP - } \\
\text { CG, CP + } \\
\text { CG, CP - }\end{array}$ & $\begin{array}{r}73 \cdot 4 \\
2 \cdot 2 \\
18 \cdot 6 \\
2.7\end{array}$ & $\begin{array}{r}85.8 \\
1.6 \\
9.8 \\
0.8\end{array}$ & $\begin{array}{r}91 \cdot 7 \\
1.3 \\
5 \cdot 3 \\
0.5\end{array}$ & $\begin{array}{r}94 \cdot 6 \\
1 \cdot 3 \\
3 \cdot 2 \\
0 \cdot 3\end{array}$ & $\begin{array}{r}95 \cdot 8 \\
1 \cdot 3 \\
2 \cdot 1 \\
0 \cdot 2\end{array}$ & $\begin{array}{r}96 \cdot 7 \\
1 \cdot 2 \\
1 \cdot 4 \\
0 \cdot 2\end{array}$ & $\begin{array}{r}97 \cdot 3 \\
1 \cdot 2 \\
1.0 \\
0 \cdot 2\end{array}$ & $\begin{array}{r}97 \cdot 7 \\
1 \cdot 1 \\
0 \cdot 8 \\
0 \cdot 1\end{array}$ & $\begin{array}{r}98 \cdot 1 \\
1 \cdot 1 \\
0 \cdot 6 \\
0 \cdot 1\end{array}$ & $\begin{array}{r}98 \cdot 3 \\
1.0 \\
0.5 \\
0.1\end{array}$ \\
\hline $\begin{array}{l}\text { Histologicall } \\
\text { CP + } \\
\text { CP - }\end{array}$ & $\begin{array}{r}\mathrm{mal} \mathrm{mu} \\
0.7 \\
2.1\end{array}$ & $\begin{array}{l}0.4 \\
1.3\end{array}$ & $\begin{array}{l}0.3 \\
0.8\end{array}$ & $\begin{array}{l}0.3 \\
0.5\end{array}$ & $\begin{array}{l}0.2 \\
0.3\end{array}$ & $\begin{array}{l}0.2 \\
0.2\end{array}$ & $\begin{array}{l}0.1 \\
0.1\end{array}$ & $\begin{array}{l}0.1 \\
0.1\end{array}$ & $\begin{array}{l}0 \cdot 1 \\
0 \cdot 1\end{array}$ & $\begin{array}{l}0.1 \\
0.0\end{array}$ \\
\hline
\end{tabular}

$\mathrm{ACG}=$ active chronic gastritis; $\mathrm{CG}=$ chronic gastritis; $\mathrm{CP}=C$ pylori

event occurs $x$ times $(x=1,2,3 \ldots n)$ is calculated using the binominal distribution.

Because there were some patients who showed $C$ pylori and active chronic gastritis in only two or three specimens, we estimated that one or two patients might have given false negative results at all biopsy sites.

The data were processed with Multiplan and Chart software (Microsoft, Aschheim, West Germany) on an IBM personal computer. Statistical tests were performed using Statgraphics software packages (STSC Rockville, Maryland, USA).

\section{Results}

Fifty unselected patients $(29 \mathrm{men} / 21$ women, aged 24-79 years, median 53 years) were examined. Thirty two patients showed active chronic gastritis at one or more of the 10 sites biopsied. Sixteen patients had chronic gastritis with no signs of activity at one or more sites; two showed completely healthy mucosa at each of the 10 biopsy sites.

When the individual biopsy sites were analysed, $C$ pylori was closely associated with active chronic gastritis. In 32 patients 234 of 320 biopsy sites showed active chronic gastritis. Two hundred and twenty seven out of 234 sites with active chronic gastritis showed $C$ pylori by the Warthin-Starry stain and 222 in the culture, respectively. These numbers were not different $(p>0.05)$ using the $\chi^{2}$ test. Warthin-Starry staining and culture were positive for $C$ pylori in 261 and 289 of 320 sites, respectively (fig 1). Compared with 234 sites showing active chronic gastritis, the area of $C$ pylori colonisation was larger $\left(\mathrm{p}<0.05, \chi^{2}\right.$ and covered the area with active chronic gastritis (fig 1).

The number of biopsy sites showing active chronic gastritis in the individual patients ranged from two sites in two patients to all sites in nine patients (fig 2 ).

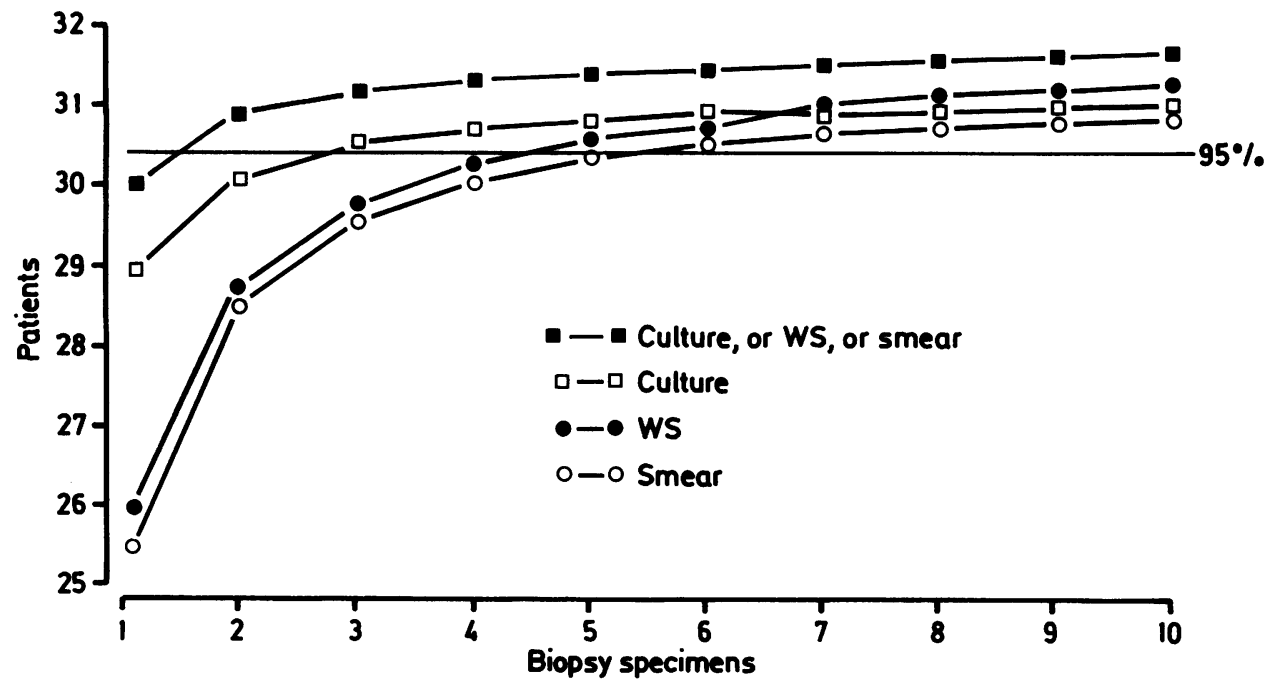

Fig 3 Sensitivity of Warthin-Starry stain, culture, and fuchsin smear for detection of $C$ pylori. The curves represent the number of patients who were $C$ pylori positive in at least one of 10 biopsy sites. 
Eight out of 32 patients with active chronic gastritis in six or fewer biopsy sites in the antrum and corpus were considered to have a patchy distribution of gastritis (fig 2).

Intestinal metaplasia was found at $\mathbf{4 2}$ sites in the 32 patients with active chronic gastritis. Thirty five out of 42 biopsy specimens showed merely circumscribed intestinal metaplasia, and only seven specimens had widespread metaplasia. One patient who was considered to have "patchy" gastritis had active chronic gastritis at four sites in the corpus and at one site in the antrum. The other biopsy sites in this patient showed widespread intestinal metaplasia. In the other seven patients intestinal metaplasia was not responsible for the patchy distribution of active chronic gastritis.

The number of biopsy specimens required to detect active chronic gastritis with haematoxylin and eosin staining, and also $C$ pylori with either Warthin-Starry stain or culture in these 32 patients with active chronic gastritis is shown in the table. Four biopsy specimens were necessary to detect active chronic gastritis with more than $95 \%$ statistical confidence, and five to detect additionally $C$ pylori by Warthin-Starry stain or by culture (table). The sensitivities of Warthin-Starry stain, culture, and smears for detecting $C$ pylori are compared in fig 3 . Initially, $C$ pylori culture had the highest sensitivity, followed by Warthin-Starry stain. In eight biopsy specimens Warthin-Starry stain was superior to culture; $C$ pylori culture missed one patient who had active chronic gastritis at only two sites. The fuchsin smear was less sensitive than Warthin-Starry stain or culture.

The association between the number of biopsy specimens and the rate of $C$ pylori positive active chronic gastritis, $C$ pylori negative active chronic gastritis, $C$ pylori positive chronic gastritis with no polymorphonuclear infiltration, and histologically normal mucosa with detection of $C$ pylori in the 32 patients with confirmed active chronic gastritis changed with increasing numbers of biopsy specimens (table). The rate of $C$ pylori positive patients with chronic gastritis decreased with the increasing number of biopsy specimens (table).

\section{Discussion}

C pylori colonisation was closely and specifically associated with active chronic gastritis. All patients with active chronic gastritis at one or more sites in the stomach were positive. $C$ pylori was not isolated from patients with only chronic gastritis, or from those with histologically healthy mucosa. A quarter of our patients showed a patchy distribution of active chronic gastritis, which agrees with the results of an earlier report. ${ }^{15}$ Taking all types of gastritis into consideration, Ottenjann et al showed that 10 out of a 100 patients showed patchy gastritis when 10 biopsies were performed and that patchy gastritis was localised in the antrum as well as in the corpus. ${ }^{\text {is }}$ Recently Hazell has suggested that four biopsy specimens are needed to detect active chronic gastritis in at least one biopsy specimen. ${ }^{10}$

Considering only the $C$ pylori state Wyatt et al found that four out of 38 patients had patchy $C$ pylori colonisation when five to eight biopsies were performed (Workshop on Gastroduodenal Pathology and $C$ pylori; Bordeaux 1988). Nedenskov et al took five biopsy specimens from the antrum and found a patchy distribution of $C$ pylori in only one out of 26 patients (Workshop on Gastroduodenal Pathology and $C$ pylori Bordeaux 1988). The results of these two studies, together with those of other authors, ${ }^{18}$ suggest that two biopsies would be sufficient to establish the C pylori state, and this has been confirmed by our data.

In agreement with Hazell, ${ }^{10}$ we found that four biopsies were needed to detect active chronic gastritis (fig 3). Widespread intestinal metaplasia was responsible for the patchy distribution of active chronic gastritis in only one patient.

The number of biopsy specimens taken greatly influenced the diagnosis of gastritis, and suggests that previously described associations of $C$ pylori and chronic gastritis may be due to the small number of biopsy specimens only from one or two sites. ${ }^{20-14}$ Our results showed that the fact that the area of $C$ pylori colonisation is more extensive than the area of active chronic gastritis in most patients (fig 1), may give rise to the misdiagnosis of $C$ pylori positive chronic gastritis in some patients (fig 2, table). The higher the number of biopsy specimens, the greater the association between $C$ pylori and active chronic gastritis, and the "association" of $C$ pylori with chronic gastritis, ${ }^{20-14}$ or $C$ pylori colonisation of histologically normal mucosa, ${ }^{12} 14$ or active chronic gastritis with no $C$ pylori colonisation, decreased. The area of colonisation by $C$ pylori was significantly larger than the area showing active chronic gastritis (fig 1), which may explain the relatively common diagnosis of $C$ pylori positive chronic gastritis. ${ }^{9-14}$ But the extent of $C$ pylori colonisation covered the area of active chronic gastritis, suggesting that $C$ pylori colonisation may precede the development of active chronic gastritis.

An analysis of individual biopsy sites showed that C pylori was also topographically associated with active chronic gastritis, even in those patients who had active chronic gastritis at only two or four sites: $\mathrm{p}<0.05$, McNemar test (fig 2). This suggests a patchy distribution of active chronic gastritis rather than methodological failure to detect either polymorphonuclear infiltration or $C$ pylori. Hazell et al suggested that $C$ pylori may be a specific marker for active chronic gastritis, but they did not show a close topographical association. ${ }^{10}$

The most important result of this study is the patchy 
distribution of active chronic gastritis, which, in fact, reduced the sensitivity of histological analysis. This patchy distribution certainly influences the specificity of serological methods, because an antibody response may also occur in patients with such a distribution. Too few biopsy specimens may produce false negative histological results in these patients and thus seemingly decrease the specificity of serological methods. ${ }^{19-23}$ Perez-Perez et al, ${ }^{20}$ taking only one antral biopsy specimen, found raised antibody titres in patients with histologically confirmed gastritis and negative $C$ pylori culture, which might be explained by a patchy distribution of $C$ pylori. Goodwin et al described a significant overlap between the antibody titres of patients with $C$ pylori positive and negative histology. ${ }^{23}$ Histological results were based on one antral biopsy specimen, suggesting that some patients had false positive serology or gave false negative results when patchy distribution of gastritis was considered. Patchy distribution of gastritis has been discussed by Wulffen et $a l^{22}$ who found raised antibody titres and $C$ pylori colonisation in patients with histologically normal antrum mucosa. They also found isolated $C$ pylori associated gastritis with an antibody response in the body of the stomach. ${ }^{22}$

Like serological studies, the sensitivity of breath tests (Ackery et al and Dill et al, Workshop on Gastroduodenal Pathology, Bordeaux, 1988) may be influenced by a patchy distribution of active chronic gastritis. Graham et al reported that $13 \%$ of his patients with duodenal ulcer had a negative breath test; others found about $98 \%$ of such patients to be colonised by $C$ pylori. ${ }^{1781324}$ It might be concluded from this result that patchy $C$ pylori colonisation in the patients produced insufficient breakdown of urea to yield a positive breath test. This would imply a much lower sensitivity of the urea breath test.

Serological tests, as well as breath test studies, have been suggested as an easy and convenient method for follow up of patients after $C$ pylori has been eradicated. We have no data as yet about the follow up of patchy gastritis. The patchy distribution of active chronic gastritis in $25 \%$ of our patients may represent an early stage of $C$ pylori infection, but it could also represent a better local host response. ${ }^{21}$ This is a matter for further investigation. Patchily distributed gastritis may develop into a diffuse distribution of gastritis, and patchy gastritis may also be the result of an incomplete eradication of $C$ pylori. Graham et $a l^{4}$ and Dill et al (Workshop on Gastroduodenal Pathology and $C$ pylori, Bordeaux, 1988) found a negative breath test in some patients who were $C$ pylori positive and had a positive breath test again when checked four weeks later immediately after bismuth treatment. This suggests that patchy gastritis must be considered when evaluating non-invasive methods, especially during follow up and in the early stages of $C$ pylori infection.

The data provided by this study suggest that five or more biopsy specimens taken anywhere in the stomach would be necessary to detect a patchy distribution of active chronic gastritis. This would seem essential, especially in serology or breath test studies in which the histological examination serves as the reference. The topographic and specific association of $C$ pylori with active chronic gastritis shown in this study further supports its pathogenic role in active chronic gastritis. The question as to whether $C$ pylori colonises healthy mucosa or previously damaged areas needs further study.

We thank professor U Stadtmüller, department of mathematics III, University of Ulm, for valuable help with the statistical analysis of the data, and the surgical department of the University of Munich for providing the software to process the data. We gratefully acknowledge the help of professor R Ottenjann, 1st medical department, Munich-Neuperlach during the preparation of the study. Dr $\mathbf{H}$ BlaufuB kindly performed the $C$ pylori cultures.

\section{References}

1 Marshall BJ, Armstrong JA, McGechie DB, Glancy RJ. Attempt to fulfill Koch's postulates for pyloric campylobacter. Med J Aust 1985;142:436-9.

2 Wyatt JI, Rathbone BJ, Heatley RV. Local immune response to gastric Campylobacter in non-ulcer dyspepsia. J Clin Pathol 1986;39:863-70.

3 Wyatt JI, Rathbone BJ, Dixon MF, Heatley RV. Campylobacter pyloridis and acid induced gastritic metaplasia in the pathogenesis of duodenitis. J Clin Pathol 1987;40:841-8.

4 Morris A, Nicholson G. Ingestion of Campylobacter pyloridis causes gastritis and raised fasting gastritic pH. Am J Gastroenterol 1987;82:192-9.

5 Bode G, Malfertheiner P, Ditschuneit $H$. Invasion of Campylobacter pylori-like organisms in the duodenal mucosa in patients with active duodenal ulcer. Klin Wosenschr 1987;65:144-6.

6 Coghlan JG, Gilligan D, Humphries H, et al. Campylobacter pylori and recurrence of duodenal ulcers-A 12 month followup-study. Lancet 1987;ii:1109-11.

7 Rauws EAJ, Langenberg W, Houthoff HJ, Zanen HC, Tytgat GNJ. Campylobacter pyloridis-associated chronic active antral gastritis. Gastroenterol 1988;94:33-40.

8 Goodwin CS, Marshall BJ, Blincow ED, Wilson DH, Blackbourn $S$, Phillips M. Prevention of nitroimidazole resistance in Campylobacter pylori by co-administration of colloidal bismuth subcitrate: clinical and in vitro studies. $J$ Clin Pathol 1988;41:207-10.

9 Glupczynski Y, Burette A, Labbe M, Deprez C, De Reuck M, Deltenre M. Campylobacter pylori-associated gastritis: a double blind placebo-controlled trial with amoxycillin. Am J Gastroenterol 1988;83:365-72.

10 Hazell SL, Hennessy WB, Borody TJ, et al. Campylobacter pyloridis gastritis II: Distribution of bacteria and associated inflammation in the gastroduodenal environment. Am J Gastroenterol 1987;82:297-301.

11 Marshall BJ, Warren JR, Francis GJ, Langton SR, Goodwin CS, Blincow ED. Rapid urease test in the management of 
Campylobacter pyloridis-associated gastritis. Am J Gastroenterol 1987;82:200-10.

12 Niedobitek F, Grosse G, Taube F, Volkheimer G, Fehrenbach FJ, Werner $E$. Investigations on the bacterial colonization of the gastric mucosa. $Z$ Gastroenterol 1987;25:98-106.

13 Hirschl AM, Stanek G, Rotter M, et al. Campylobacter pylori, gastritis and ulcus pepticum. Wien Klin Wochenschr 1987;99:493-7.

14 Anderson LP, Holck S, Povlson CO, Elsborg L, Justesen T. Campylobacter pyloridis in peptic ulcer disease. Scand $J$ Gastroenterol 1987;22:219-24.

15 Ottenjann R, Rösch W, Elster K. Is gastritis a diffuse process? Klin Wosenchr 1971;49:27-31.

16 Kasper G, Dickgiesser N. Isolation of campylobacter-like bacteria from gastric epithelium. Infection 1984;12:179-80.

17 Whitehead R, Truelove SC, Gear MNL. The histological diagnosis of chronic gastritis in fibre optic gastroscope biopsy specimens. J Clin Pathol 1972;25:1-11.

18 Börsch G, Adamek R, Sandmann M, Wegener M, Schmidt G, Leverkus F, Reitemeyer E. Comparison of biopsy urease test and histologic examination for detection of Campylobacter pylori. Hepatogastroenterol 1987;34:236-41.

19 Rathbone BJ, Wyatt JI, Worsley BW, Shires SE, Trejdosiewicz LK, Heatley RV, Losowsky MS. Systemic and local antibody responses to gastric Campylobacter pyloridis in non-ulcer dyspepsia. Gut 1986;27:642-7.
20 Perez-Perez GI, Dworkin BM, Chodos JE, Blaser MJ. Campylobacter pylori antibodies in humans. Ann Intern Med 1988;109:11-7.

21 von Wulffen H, Grote HJ. Enzyme-linked immunosorbent assay for detection of immunoglobulin $A$ and $G$ antibodies to Campylobacter pylori. Eur J Clin Microbiol 1988;7:559-65.

22 von Wulffen H, Grote HJ, Gatermann S, Löning T, Berger B, Buhl C. Immunoblot analysis of immune response to Campylobacter pylori and its clinical associations. J Clin Pathol 1988;41:653-9.

23 Goodwin CS, Blincow E, Peterson G. Enzyme-linked immunosorbent assay for Campylobacter pyloridis: Correlation with presence of $C$. pyloridis in the gastric mucosa: $J$ Infect Dis 1987;155:488-94.

24 Graham DY, Klein PD, Evans DJ Jr, et al. Campylobacter pylori detected non-invasively by the ${ }^{13} \mathrm{C}$-urea breath test. Lancet 1987;i:1174-7.

Address for reprints to: Dr E Bayerdörffer, Medical Department II, Klinikum Großhadern, Marchioninistr: 15, D-8000 Munich 70, FRG. 